\title{
Narrow-band ultraviolet B (NB UV-B) exposures improve mood in healthy individuals differently depending on chronotype
}

Toledo Anna,,${ }^{1,2}$ Karppinen Toni, ${ }^{1,2}$ Miettinen Maija E, ${ }^{3}$ Leppäluoto Juhani, ${ }^{4}$ Vuolteenaho Olli, ${ }^{4}$ Ylianttila Lasse, ${ }^{5}$ Kautiainen Hannu, ${ }^{6}$ Snellman Erna, ${ }^{1,2}$ Partonen Timo ${ }^{3}$

${ }^{1}$ Tampere University, Faculty of Medicine and Health Technology, Department of Dermatology and Venereology. Tampere, Finland

${ }^{2}$ Tampere University Hospital, Department of Dermatology and Allergology. Tampere, Finland

${ }^{3}$ National Institute for Health and Welfare (THL), Department of Public Health Solutions. Helsinki, Finland

${ }^{4}$ University of Oulu, Department of Physiology. Oulu, Finland

${ }^{5}$ STUK - Radiation and Nuclear Safety Authority. Helsinki, Finland

${ }^{6}$ Unit of Primary Health Care, Helsinki University Central Hospital, Department of General Practice, University of Helsinki, and Unit of Primary Health Care, Kuopio University Hospital. Helsinki and Kuopio, Finland

Word count: 4251

Figures: 2

Tables: 1

Abbreviations: ultraviolet radiation (UVR), narrow-band UV-B (NB UV-B), Visual Analogue Scales (VAS), $\beta$-endorphin ( $\beta$-END), Interleukin-6 (IL-6)

\section{Corresponding author:}

Anna Toledo, Tampere University Hospital,

PL 2000,

33521 Tampere, Finland

+3583311611

anna.toledo@tuni.fi 


\section{Abstract}

The evening chronotype is associated with psychological symptoms such as depressed mood, while skin exposure to ultraviolet radiation (UVR) may affect mood and behavior through neural and humoral routes. This pilot study aimed to investigate the impact of whole-body narrow-band (NB) UV-B exposure on current mood state and circulating 25-hydroxyvitamin D3 (25(OH)D $\mathrm{D}_{3}$, interleukin-6 (IL-6), cortisol and $\beta$-endorphin ( $\beta$-END) levels in healthy participants. Here, eleven healthy women received full-body NB UV-B exposures on four afternoons, and the chronotype was assessed with a shortened version of Horne and Östberg's Morningness-Eveningness Questionnaire (MEQ). Perceived mood was evaluated using the Visual Analogue Scale (VAS), and serum 25(OH) $\mathrm{D}_{3}$, IL-6, cortisol and $\beta$-END concentrations were monitored daily. Decreasing VAS values showed mood to improve significantly over the five days after the four suberythematous NB UV-B exposures ( $p=0.038)$, and the more the circadian preference was inclined towards eveningness, the greater the improvement in the mood dimension of wellbeing $(\mathrm{p}=0.021)$. Baseline mood state was correlated with baseline $25(\mathrm{OH}) \mathrm{D}_{3}(\mathrm{r}=-0.54$, 95\% CI: -0.86 to -0.09$)$ and with baseline cortisol ( $r=-0.57,95 \%$ CI: -0.87 to -0.04$)$. During the NB UV-B exposures, $25(\mathrm{OH}) \mathrm{D}_{3}$ increased significantly, as expected, and IL-6 declined significantly by -0.35 (95\% CI: -0.69 to -0.07$) \mathrm{pg} / \mathrm{mL}$ from the initial values of $1.12 \pm 0.66 \mathrm{pg} / \mathrm{mL}(\mathrm{p}=0.025)$. In conclusion, in our pilot study, NB UV-B exposure improved mood, especially among those with evening preference for their daily activities, as well as circulating $25(\mathrm{OH}) \mathrm{D}_{3}$ levels, whereas circulating IL-6 levels decreased.

Keywords: Chronotype; mood; Vitamin D; $\beta$-endorphin; cortisol; interleukin-6; UV-B 


\section{Introduction}

Chronotype is a measure of individual differences in daily activity patterns and sleep-wake cycles whereby humans can be classified into three chronotypes based on the timing of physiological functions and their preference in daily activities: morning types (advanced sleep period), neither types, and evening types (delayed sleep period) (Duffy et al. 2001; Horne and Östberg 1976). The chronotype has a genetic basis and is influenced by environmental factors such as latitude and photoperiod (Randler and Rahafar 2017; Shawa et al. 2018). Higher latitudes are characterized by greater seasonal variations in photoperiod, and people living at high latitudes seem to have a tendency towards a late time of day preference (Borisenkov et al. 2010; Leocadio-Miguel et al. 2017). Previous studies suggest that the preference for eveningness may be associated with depressive symptoms and mood disorders (Au and Reece 2017; Hidalgo et al. 2009; Jeong Jeong et al. 2015; Merikanto et al. 2013).

Circadian rhythms are present in near all tissues throughout the human body, and the circadian system is responsible for regulating the approximate 24-hour rhythms in biological (e.g., hormone levels), psychological (e.g., alertness and mood) and behavioral (e.g., eating, exercising and sleeping) variables (Adan et al. 2012; Dibner 2019; Lemmer 2009). The individual morningness-to-eveningness preferences arise from the differences in the endogenous circadian clock and the daily schedule for activities as well as the interplay between these two systems (Barclay et al. 2014; Dijk and von Schantz 2005; Kerkhof 1985). Circadian rhythms are generated and maintained by the intrinsic clocks to track time and correspond to the temporal organization of the environment. Light exposure is the most potent time cue for synchronizing internal circadian rhythms with the transitions of the external light-dark cycle (Münch and Bromundt 2012; Roenneberg et al. 2007; Zeitzer et al. 2005). Human skin is repeatedly exposed to solar ultraviolet radiation (UVR), a confirmed environmental carcinogen that induces mutations and simultaneous immunosuppression (Hart 
and Norvall 2018; Murphy 2009; World Health Organization 2012). The ultraviolet B (UV-B) wavebands (315-280 $\mathrm{nm}$ ) are biologically the most active part of the solar spectrum reaching the Earth, and UV-B-induced DNA damage leads to the release of various pro-inflammatory mediators from keratinocytes, including interleukin-6 (IL-6) (Hart and Norvall 2018; Murphy 2009; Urbanski et al. 1990; World Health Organization 2012). In addition, UV-B radiation exerts strong biological actions on regulatory pathways related to immune balance, both vitamin-D-related and vitamin-D-independent (Skobowiat and Slominski 2015).

UV-B radiation induces the cutaneous photosynthesis of vitamin D, which leads to increased levels of its circulating form, i.e., 25-hydroxyvitamin D3 (25(OH) $\left.\mathrm{D}_{3}\right)$. Vitamin D insufficiency (levels of 50-75 nmol/L) and deficiency (levels of $<50 \mathrm{nmol} / \mathrm{L}$ ) are common at high latitudes in winter unless vitamin D substitution is used (Holick 2007; Holick and Chen 2008). Low $25(\mathrm{OH}) \mathrm{D}_{3}$ status has been linked to poorer mental health and may explain the low vitality, energy and mood observed in winter (Berk et al. 2007; Harris and Dawson-Hughes 1993; Humble 2010; Jääskeläinen et al. 2015).

IL-6 and cortisol are first-line immune modulators that can be synthesized in the skin under stress conditions, including UVR exposure, and delivered in the circulation (Kirnbauer et al. 1991; Skobowiat et al. 2011; Skobowiat and Slominski 2015; Urbanski et al. 1990). Elevated IL-6 levels have been detected in patients presenting with major depressive disorder (Hodes et al. 2016; Liu et al. 2012), while cortisol has been associated with the immune system and mood (Shattuck and Muehlenbein 2015). Patients who did not respond and were resistant to antidepressant medications had increased circulating TNF- $\alpha$ and IL-6 levels and impaired cutaneous glucocorticoid receptor function (Fitzgerald et al. 2006). A large proportion of depressed patients showed excessive cortisol production (Carpenter and Gruen 1982).

Beta-endorphin ( $\beta$-END) is formed in the epidermal skin from proopiomelanocortin (POMC) upon UVR exposure (Fell et al. 2014; Skobowiat and Slominski 2015). Human studies of 
UVR-induced $\beta$-END are rare, however, and the results are conflicting. In mice, UV-Binduced $\beta$-END led to the development of UVR-addictive behavior, and it has been suggested that it may underlie tanning-dependent behavior in humans (Skobowiat and Slominski 2015).

This pilot study investigated the impact of whole-body narrow-band (NB) UV-B exposure on the current mood state, in general and according to chronotype, in healthy participants and the associations with the circulating 25(OH) $\mathrm{D}_{3}$, IL-6, cortisol and $\beta$-END levels. We hypothesized that a) NB UV-B exposure improves the current mood state and that b) this improvement occurs through the skin-borne circulating mediators and is reflected in changes in their circulating levels. Further, we hypothesized that these changes are greater in individuals with an eveningness preference for daily activities because a) they tend to have a higher ratio of phase advancing to phase delaying of circadian rhythms by light exposure (Emens et al. 2009) and b) they appear to benefit from the phase advance accompanied by improvements in self-reported depression and stress (Facer-Childs et al. 2019).

\section{Materials and Methods}

The study was implemented at the Department of Dermatology and Venereology, Tampere University Hospital, Tampere, Finland, $61^{\circ} \mathrm{N}$, in October to November, when the availability of biologically effective skin-burning UV-B solar ultraviolet radiation was negligible. The protocol followed the principles of the Declaration of Helsinki and its amendments and was approved by the Regional Ethics Committee of Tampere University Hospital District (\# R14012). The volunteers were recruited through advertisements and provided informed consent. No compensation was given.

\section{Volunteers}

Twelve healthy women agreed to participate. The inclusion criteria were as follows: a) they had no phototherapy, solarium visits or sunny holidays during the preceding two months, and 
b) they had taken no vitamin D supplements during the preceding two months. The volunteers had to regard themselves as "healthy” and have not had any psychiatric interventions. Depression was not specifically screened. The exclusion criteria were a) sun-sensitive Fitzpatrick skin phototype I, b) the use of antidepressants or any psychotropic drugs, c) the use of medication with definite photosensitizing potential (specifically, amiodarone, dacarbazine, doxycycline, phenothiazine, furosemide, diclofenac, NSAIDs, ketoprofen, and piroxicam) and d) pregnancy. We also asked whether the volunteer was using any medication, and if so, we asked them to name it. Demographic data were collected with respect to age, gender, skin phototype (Fitzpatrick 1988) and body-mass index (BMI).

\section{Narrow-band UV-B exposures}

The volunteers received four full-body NB UV-B exposures in the afternoons of four consecutive days. Their eyes were covered with opaque goggles during the irradiations. The irradiance of the Waldmann UV 7002 phototherapy cabin (Herbert Waldmann GmbH \& Co. KG, Villingen-Schwenningen, Germany), which was equipped with 42 TL01 tubes (Schulze \& Böhm GmbH, Brühl, Germany), was measured beforehand using an Ocean Optics S2000 spectroradiometer (Ocean Optics, Inc., Largo, FL, USA). After correcting for systematic errors and stray light, it was estimated that the uncertainty $(2 \sigma)$ of the measurement was approximately 14\%. These assessments were traceable to the National Institute of Standards and Technology (Gaithersburg, MD, USA). The initial NB UV-B physical dose was 0.17 $\mathrm{J} / \mathrm{cm}^{2}$, corresponding to one standard erythema dose (1 SED), which is equivalent to an erythemal effective radiant exposure of $10 \mathrm{~mJ} / \mathrm{cm}^{2}$ (Commission Internationale de l'Éclairage 1999). In the course of the four days, the dose was increased from $0.17 \mathrm{~J} / \mathrm{cm}^{2}$ to $0.34 \mathrm{~J} / \mathrm{cm}^{2}$ (2 SED) for 5 volunteers and to $0.51 \mathrm{~J} / \mathrm{cm}^{2}$ (3 SED) for 6 volunteers. The cumulative dose of the four irradiations thus ranged from $1.19 \mathrm{~J} / \mathrm{cm}^{2}$ (7 SED) to $1.36 \mathrm{~J} / \mathrm{cm}^{2}$ (8 SED).

\section{Evaluation of mood state}


To determine whether the four NB UV-B exposures affected the participants' mood state, they were asked to rate their mood on each day immediately before the scheduled NB UV-B exposure and on day five 24 hours after the last exposure, employing the Visual Analogue Scale (VAS), where values closer to zero depict better feelings (Folstein and Luria 1973). The eventual mood VAS used in the statistical analyses was the mean of four dimensions of mood, 1) VAS-satisfaction, 2) VAS-tiredness, 3) VAS-wellbeing and 4) VAS-irritation, each scored separately (in millimeters) on a 100-mm line with its ends anchored to the concepts of “best ever” versus “worst ever”. The questions above the VAS scales asked "How do you rate your feelings of satisfaction/tiredness/wellbeing/irritation at the moment?”

\section{Morningness-Eveningness Questionnaire (MEQ-6)}

A Morningness-Eveningness Questionnaire (MEQ-6, v.1.2 FI, National Institute for Health and Welfare, Helsinki, Finland) based on the original Horne \& Östberg MorningnessEveningness (MEQ-19) questionnaire translated to Finnish was used to define the chronotype (Horne and Östberg 1976). The modified instrument comprises six questions. All the answers were scored and summed for the total Morningness-Eveningness score of 5 to 27 points. In our analyses, the participants were classified into morning or evening chronotypes ("Larks” vs. “Owls”) based on the last question in MEQ-6, where the definitely morning and rather morning than evening chronotypes were classified as "Larks", and definitely evening and rather evening than morning chronotypes were classified as “Owls”.

\section{Time schedule for blood sampling}

Blood samples were taken prior to each NB UV-B exposure to measure circulating $25(\mathrm{OH}) \mathrm{D}_{3}$, IL-6, cortisol and $\beta$-END levels. All samples were drawn at the same hour of the afternoon on five consecutive days. The first sample was taken on day 0 before the first exposure, and the last sample was taken 24 hours after the final NB UV-B exposure. The 
blood samples were protected from the light and centrifuged, whereupon the sera were separated out and stored at $-70^{\circ} \mathrm{C}$ until used for the analyses.

\section{Laboratory analyses}

25(OH) $\mathrm{D}_{3}$ was determined by a Chemiluminescent Microparticle Immunoassay using the Architect system (Abbott Laboratories, Abbott Park, IL, USA). Quality control was further ensured by participation in the International External Quality Assessment Scheme (DEQAS) (Miettinen et al. 2014). Due to the short duration of the trial, no nutritional analysis of the availability of vitamin D in the diet was performed, but the volunteers were asked to keep their diet constant during the experiment.

Serum immunoreactive $\beta$-END was measured using an ELISA method developed at the University of Oulu, Finland. Samples and synthetic human beta-endorphin calibrators were incubated with synthetic beta-endorphin-containing C-terminal biotin and the rabbit antihuman beta-endorphin antiserum "BK22” (Vuolteenaho 1984) at a final dilution of 1/100000. After overnight incubation at $+4^{\circ} \mathrm{C}$, streptavidin-horseradish peroxidase conjugate (Pierce, Thermo Fisher Scientific Inc., Waltham, MA, USA) was added, followed by 3,3',5,5'tetramethylbenzidine substrate (TMB One, KemEnTec Diagnostics A/S, Taastrup, Denmark). The reaction was stopped with 2-N sulfuric acid, and the wells were scanned at $\mathrm{A}_{450 \mathrm{~nm}}$ with a Varioskan Flash Plate Reader (Thermo Scientific, Thermo Fisher Scientific Inc., Waltham, MA, USA). Serum cortisol was measured using a Ref 52611 ELISA kit from IBL International GmbH, Hamburg, Germany, and IL-6 was measured with a DY206 ELISA kit from R\&D Systems, Inc., Minneapolis, MN, USA, according to the instructions provided by the manufacturers.

\section{Statistical analyses}


We report the results as the means with standard error of mean (SEM) and 95\% confidence intervals (CIs). Due to the sample size and some skewed distributions, resampling-based (Bootstrap or Monte-Carlo) methods were used in the analyses to define the significance level (p-value) and 95\% CIs. Mean changes (within subjects) in mood and physiological biomarkers between day 5 and baseline values were assessed using the Fisher-Pitman permutation test for paired replicates (exact p-value). Changes on day 5 and baseline values between chronotypes were compared using bootstrap-type independent-sample t-tests (5000 replications). Statistical significance for the hypothesis of linearity across the 5-day periods of biomarkers was tested using bootstrap-type repeated-measures analysis of variance (rANOVA). Effect size (d) was calculated using the method of Cohen, where an effect size of 0.20 is considered small, 0.50 moderate, and 0.80 large; CIs for the effect sizes were obtained by bias-corrected bootstrapping (5000 replications). We used linear regression models to evaluate the contributions of the total Morningness-Eveningness score and the skin phototype as independent predictors of the dependent outcome of the change in VAS-wellbeing. We calculated the correlation coefficients using Spearman's method. The normality of the variables was tested using the Kolmogorov-Smirnov test. No adjustment was made for multiple testing, but this information can be obtained by multiplying the actual p-value by the number of comparisons made. Statistical analyses were carried out using the Stata statistical software, release 14.1 (StataCorp, College Station, TX, USA), and the IBM Statistical Package for the Social Sciences (SPSS) Statistics, version 25 (International Business Machines Corporation, Armonk, NY, USA).

\section{Results}

\section{Chronotype and other background characteristics}

Eleven of 12 women completed the experiment, since one withdrew for personal reasons. Five volunteers had a preference towards morningness (Larks) and six towards eveningness 
(Owls). The mean age was $41.6 \pm 11.2$ years. Eight volunteers $(72.7 \%)$ displayed skin phototype II (often burns and tans poorly), and three (27.3\%) displayed skin phototype III (sometimes burns and tans easily) (Fitzpatrick 1988). The mean BMI was $25.1 \pm 5.1 \mathrm{~kg} / \mathrm{m}^{2}$.

\section{Impact of NB UV-B exposures on mood}

The mean VAS score (VAS-mean) of the eleven participants on the four mood dimensions (satisfaction, tiredness, wellbeing and irritation) was 28.6 $\pm 15.9 \mathrm{~mm}$ before the NB UV-B exposures, which improved between day 5 and baseline significantly by a mean of 7.4 (95\% CI: -14.5 to -0.3$) \mathrm{mm}$ by the end of the study ( $\mathrm{p}=0.038$, Figure 1$)$. The declining VAS-mean depicted improved mood in eight of eleven participants (73\%), five of whom were Owls. The VAS-mean was higher (i.e., the mood was worse) in the Owls than in the Larks on each day. On the other hand, the VAS-mean decreased from baseline to day 5 in the Owls, 11.8 (95\% CI: -21.4 to -2.2) mm, and the Larks, 2.1 (95\% CI: -11.6 to 7.5$) \mathrm{mm}$, i.e., the mood state improvement was larger in the Owls, but the difference between the groups was not statistically significant $(\mathrm{p}=0.19)$ (Figure 1 and Table 1$)$.

There was a difference in the change in the "VAS-wellbeing" mood dimension from baseline to day 5 between the Larks [1.0 (95\% CI: -5.6 to 7.6)] and Owls [-16.8 (95\% CI: -25.8 to 5.8)] amounting to -17.8 (95\% CI: -31.92 to -3.75$) \mathrm{mm}$ on average ( $\mathrm{p}=0.019)$. Furthermore, in the regression analysis, the total Morningness-Eveningness score alone significantly explained the change in VAS-wellbeing $\left(\mathrm{R}^{2}=0.47 ; \mathrm{B}=-2.10,95 \% \mathrm{CI}\right.$ : -3.80 to -0.40 ; $\mathrm{p}=0.021$ ), whereas the skin phototype alone did not. However, the model (with $\mathrm{R}^{2}=0.72$ ), in which we entered both the total Morningness-Eveningness score $(B=-2.54,95 \%$ CI: -3.92 to -1.15; $\mathrm{p}=0.003)$ and the skin phototype $(\mathrm{B}=-15.34,95 \% \mathrm{CI}:-28.59$ to $-2.10 ; \mathrm{p}=0.028)$, explained significantly the change in VAS-wellbeing, and showed greater improvement among the Owls and among those having skin phototype II. Adding age to this model $\left(\mathrm{R}^{2}=\right.$ 0.85) yielded similar results and pointed to the same two predictors as being significant. 


\section{Impact of UVR irradiations on circulating biomarkers}

The baseline 25(OH) $\mathrm{D}_{3}$ values ranged from 43 to $128 \mathrm{nmol} / \mathrm{L}$, with two volunteers apparently being vitamin-D-deficient (below $50 \mathrm{nmol} / \mathrm{L}$ ) and three displaying vitamin D insufficiency (levels 50-75 nmol/L) (Holick 2007; Holick and Chen 2008). The four NB UV-B whole-body exposures effectively improved the $25(\mathrm{OH}) \mathrm{D}_{3}$ levels of all 11 volunteers by 8.3 (95\% CI: 5.6 to 11.8$) \mathrm{nmol} / \mathrm{L}$ from the initial values of $75.5 \pm 28.0 \mathrm{nmol} / \mathrm{L}(\mathrm{p}<0.001$; effect size 1.50 (95\% CI: 0.69 to 2.30)) (Figure 2a), and increases seemed detectable as early as after the first NB UV-B exposure. The increase was greatest in the volunteers with the lowest baseline values. None of the women were vitamin-D-deficient any longer by day five, but the five with the lowest baseline 25(OH)D 3 levels remained vitamin-D-insufficient (Holick 2007; Holick and Chen 2008).

The initial IL-6 values ranged from 0.34 to $2.21 \mathrm{pg} / \mathrm{mL}$, showing a 7-fold interindividual variation, but the mean value seemed to decrease significantly by -0.35 (95\% CI: -0.69 to 0.07) $\mathrm{pg} / \mathrm{mL}$ from the initial level of $1.12 \pm 0.66 \mathrm{pg} / \mathrm{mL}(\mathrm{p}=0.025$; effect size 0.54 (95\% CI: 0.09 to 1.31$)$ ) (Figure $2 b)$.

Baseline cortisol values varied from 243 to $526 \mathrm{nmol} / \mathrm{L}$, and among volunteers, the mean level had increased by 79 (95\% CI:-18 to 177$)$ nmol/L from the initial level of $391 \pm 81 \mathrm{nmol} / \mathrm{L}$ following the NB UV-B irradiations (Figure 2c), although this difference was not statistically significant $(\mathrm{p}=0.16)$.

The baseline $\beta$-END values showed a notable 45 -fold interindividual variation, ranging from 18 to $362 \mathrm{fmol} / \mathrm{mL}$ giving an initial mean of $203 \pm 129 \mathrm{fmol} / \mathrm{mL}$. This increased by 39 (95\% CI: -50 to 129) fmol/mL following the NB UV-B exposures, but the difference was not statistically significant $(\mathrm{p}=0.41) . \beta$-END increased in six volunteers and decreased in five volunteers over the five days of the experiment (Figure 2d). 
The mean serum $25(\mathrm{OH}) \mathrm{D}_{3}, \beta$-END, cortisol and IL-6 levels on each day are shown by chronotype (Larks/Owls) in Table 1. The mean 25(OH)D $\mathrm{D}_{3}$ levels were lower in the Owls throughout, as were the mean IL-6 levels. Mean $\beta$-END seemed higher in the Owls than in the Larks at the end of the study, but there was considerable overall fluctuation in both groups. Mean cortisol levels seemed to be higher in the Owls on each day (Table 1).

\section{Correlations between perceived mood and circulating biomarkers}

Although the volunteers' baseline 25(OH) $\mathrm{D}_{3}$ levels correlated with the baseline mood state (VAS mm) ( $r=-0.54,95 \%$ CI: -0.86 to $-0.09, p=0.085)$, the improvement in $25(\mathrm{OH}) \mathrm{D}_{3}$ did not correlate with the improvement in mood state on day five $(r=-0.14,95 \%$ CI: -0.68 to $0.50, \mathrm{p}=0.66)$.

The baseline IL-6 (pg/mL) values and perceived mood state (VAS mm) were not correlated (r $=0.06,95 \%$ CI: -0.56 to $0.64, p=0.85$ ), nor were the NB UV-B-induced increase in IL-6 and the improvement in mood ( $r=-0.44,95 \% \mathrm{CI}:-0.82$ to $0.22, \mathrm{p}=0.18)$, but the individual volunteers with higher baseline IL-6 clearly displayed a better mood state.

The volunteers' cortisol levels at baseline correlated with their mood state $(r=-0.57,95 \%$ CI: -0.87 to $-0.04, \mathrm{p}=0.065)$, but there was no correlation between the subsequent UVR-induced increase in cortisol and the improvement in the mood VAS result ( $r=-0.03,95 \%$ CI: -0.62 to 0.58, $\mathrm{p}=0.94)$.

Initial $\beta$-END values and mood VAS were not correlated $(r=0.43,95 \%$ CI: -0.23 to 0.82 , $\mathrm{p}=0.18$ ), nor was there any correlation between the increase detected in $\beta$-END and the improvement in mood VAS ( $\mathrm{r}=-0.15$, 95\% CI: -0.69 to 0.49 , $\mathrm{p}=0.65$ ).

\section{Discussion}

Our findings suggest a relationship between NB UV-B exposure and the mood state of the volunteers, referring to the fact that the improvement in mood was greater in those with their 
preference towards eveningness rather than morningness, the effect being significant for the change in the VAS-wellbeing dimension. NB UV-B significantly improved the balance of circulating vitamin D, and circulating IL-6 levels seemed to decrease. These findings suggest that further studies are required on the interactions between UVR, skin and mood.

There is evidence of an association between chronotype and mood disorders, as those with a circadian preference favoring eveningness appear to be prone to depression (Au and Reece 2017; Hidalgo et al. 2009; Jeong Jeong et al. 2015; Merikanto et al. 2013). In previous studies, mood assessments were used to evaluate depression or depressive symptoms over a longer period of time, e.g., during the past 12 months. In the present study, the volunteers who reported that they were evening chronotypes had a worse current mood state on average than the others throughout the five-day trial. The evening chronotypes also tended to show more improvement in their current mood state than the morning chronotypes following UVR exposure. However, because the participants in the current study were healthy, and as depression was not specifically screened and interviewed by us, any comparison between the current study and past research should be made with caution. To extend and deepen the scope of such generalizations, further studies need to be focused on volunteers with a current depressive episode, as assessed using a structured clinical interview for diagnosis, to elucidate the effects and mechanisms of action of NB UV-B exposures on mood. Future studies are required to show the possible causal relationship between depressive symptoms and evening chronotype and, further, whether it could be worthwhile trying NB UV-B phototherapy for people suffering from depressive symptoms or other mood disorders.

The skin is capable of reacting to psychiatric and psychosocial conditions and is a potential source of numerous UVR-inducible neuroactive compounds (Denda et al. 2013; Slominski et al. 2012; Slominski and Wortsman 2000). We have shown earlier that NB UV-B exposure increases the expression of $\beta$-END in the human skin in vivo (Jussila et al. 2016), and mouse 
experiments have shown UV-B exposure to be capable of inducing $\beta$-END expression in the skin even to the point of developing addictive behavior towards UV radiation (Fell et al. 2014; Skobowiat and Slominski 2015). In the present study, the circulating $\beta$-END levels were increased during NB UV-B exposures, although not significantly, as there was up to 45fold interindividual variation in these levels.

The present study demonstrated that the skin phototype together with the chronotype contributed significantly to the improvement in mood as assessed by means of scores on the subscale dimension of wellbeing on the VAS scales. The improvement was greater in the participants with skin phototype II than in those with skin phototype III. Phototype II burns more easily and tans less well than phototype III (Fitzpatrick 1988). Fair-skinned people are known to be more sensitive to the effects of UVR, which could explain the better moodenhancing property of the UV-B exposures in our study. It has been reported in an earlier review that UVR was found to be capable of improving mood in six out of seven existing studies (Veleva et al. 2018). An explanation here might be that NB UV-B exposure influences mood and behavior through, e.g., thermoregulation, which in depressed individuals is often disturbed. The tentative warming of the body during UV-B exposure might interfere with mood, as is shown for those with major depressive disorder, whereas warming with infrared light produces therapeutic benefits, which could occur through the deactivation of brown adipose tissue (Janssen et al. 2016).

We found that vitamin D status was lower in those volunteers who reported that their daytime peak was mainly in the evening rather than in the morning. One earlier study has shown that evening types have unhealthier dietary habits and that they ingest less vitamin D than morning types (Kanerva et al. 2012). To test the hypothesis that NB UV-B-induced increases in circulating vitamin D levels are associated with improved mood, much further study is necessary. 
Four NB UV-B exposures with low nonerythematous physiological dosing also induced a significant decrease in IL-6 levels, and we also noted that the volunteers with evening preference displayed lower IL-6 levels throughout the study than those with morning preference. In an earlier study, Mondin et al. (2016) discovered that healthy individuals with a reversed day/night schedule had decreased IL-6 levels. In contrast to our findings, Urbanski et al. (1990) showed an increase in IL-6 levels after broadband (BB) UV-B exposure, with a peak value at 12 hours, but the BB UV-B doses used were aggressively high. However, further studies are necessary to verify any connection between UV-B exposure-induced IL-6 levels and mood.

We detected that UV-B seemed to induce an increase in cortisol levels, although not to a significant extent in our pilot study, and the average cortisol levels were slightly higher in the evening types than in the morning types on each day. Both UV-B and UV-C wavelengths have been shown previously to elevate cortisol levels in the skin (Fitzgerald et al. 2006). In the case of our study, the taking of the blood samples in the afternoon instead of the morning may be reflected in a decreased response, and this should be taken into account in further studies.-We did expect that the circulating mediators of the NB UV-B exposures we assessed would correlate with the perceived changes in the current mood state, but they did not show any correlation with mood.

The limitations of our pilot study included the small number of volunteers, only female volunteers (since no men volunteered), and the lack of a control group. The groups of each chronotype were small, and some findings showed trends rather than reaching significance. Moreover, all the volunteers were healthy.

\section{Conclusions}

Our results indicate that four suberythematous exposures to NB UV-B can induce a change in perceived mood and 25(OH)D $\mathrm{D}_{3}$ as well as IL-6 levels. Mood improved more in evening 
chronotypes than morning chronotypes. Our findings support the hypothesis that UVRexposed human skin transmits cues via reciprocal molecular interactions that affect mood regulation in the brain and contribute to behavior and the timing of daily activities. However, the demonstration of the exact mechanisms remains a major challenge.

\section{Acknowledgments}

This study was supported financially by Competitive State Research Financing for this expert responsibility area of Tampere University Hospital. The authors AT and TK implemented the study; MEM, JL and OV performed the biochemical analyses; LY performed the UVR dosimetry; HK performed the statistical analyses; and all the authors took part in writing the manuscript.

\section{Disclosure of interest}

The authors report no conflicts of interest.

\section{Funding sources}

This work was supported by Competitive State Research Financing, grants \#9P065 and \#9T061, within the relevant expert responsibility area of Tampere University Hospital.

\section{References}

1. Adan A, Archer SN, Hidalgo MP, Di Milia L, Natale V, Randler C. 2012. Circadian typology: a comprehensive review. Chronobiol Int. 29:1153-75.

2. Au J, Reece J. 2017. The relationship between chronotype and depressive symptoms: A meta-analysis. J Affect Disord. 15:93-104.

3. Barclay NL, Watson NF, Buchwald D, Goldberg J. 2014. Moderation of genetic and environmental influences on diurnal preference by age in adult twins. Chronobiol Int. 31:222-31.

4. Berk M, Sanders KM, Pasco JA, Jacka FN, Williams LJ, Hayles AL, Dodd S. 2007. Vitamin D deficiency may play a role in depression. Med Hypotheses. 69:1316-19. 
5. Borisenkov MF, Perminova EV, Kosova AL. 2010. Chronotype, sleep length, and school achievement of 11-23-year-old students in northern European Russia. Chronobiol Int. 27:1259-70.

6. Carpenter WT Jr, Gruen PH. 1982. Cortisol's effects on human mental functioning. J ClinPshychopharmacol. 2:91-101.

7. Commission Internationale de l'Éclairage (CIE). 1999. Erythemal reference action spectrum and standard erythemal dose. CIE standard ISO 17166:1999(E) CIE S 007/ E 1998.

8. Denda M, Takei K, Denda S. 2013. How does epidermal pathology interact with mental state? Med Hypotheses. 80:194-6.

9. Dibner C. 2019. The importance of being rhythmic: Living in harmony with your body clocks. Acta Physiol. 12:e13281.

10. Dijk DJ, von Schantz M. 2005. Timing and consolidation of human sleep, wakefulness, and performance by a symphony of oscillators. J Biol Rhythms. 20:27990.

11. Duffy JF, Rimmer DW, Czeisler CA. 2001. Association of intrinsic circadian period with morningness-eveningness, usual wake time, and circadian phase. BehavNeurosci. 115:895-9.

12. Emens JS, Yuhas K, Rough J, Kochar N, Peters D, Lewy AJ. 2009. Phase angle of entrainment in morning- and evening-types under naturalistic conditions. Chronobiol Int. 26:474-93.

13. Facer-Childs ER, Middleton B, Skene DJ, Bagshaw AP. 2019. Resetting the late timing of 'night owls' has a positive impact on mental health and performance. Sleep Med. 60:236-47.

14. Fell GL, Robinson KC, Mao J, Woolf CJ, Fisher DE. 2014. Skin $\beta$-endorphin mediates addiction to UV light. Cell. 57:1527-34.

15. Fitzgerald P, O’Brien SM, Skully P, Rijkers K, Scott LV, Dinan TG. 2006. Cutaneous glucocorticoid receptor sensitivity and pro-inflammatory cytokine levels in antidepressant-resistant depression. Psychol Med. 36:37-43.

16. Fitzpatrick TB. 1988. The validity and practicality of sun-reactive skin types I through VI. Arch Dermatol. 124:869-71.

17. Folstein MF, Luria R. 1973. Reliability, validity, and clinical application of the visual analogue mood scale. Psychol Med. 3:479-86.

18. Harris S, Dawson-Hughes B. 1993. Seasonal mood changes in 250 normal women. Psychiatry Res. 49:77-87. 
19. Hart PH, Norvall M. 2018. Ultraviolet radiation-induced immunosuppression and its relevance for skin carcinogenesis. PhotochemPhotobiol Sci. 17:1872-84.

20. Hidalgo MP, Caumo W, Posser M, Coccaro SB, Camozzato AL, Chaves ML. 2009. Relationship between depressive mood and chronotype in healthy subjects. Psychiatry ClinNeurosci. 63:283-90.

21. Hodes GE, Ménard C, Russo S. 2016. Integrating Interleukin-6 into depression diagnosis and treatment. Neurobiol Stress. 4:15-22.

22. Holick MF. 2007. Vitamin D deficiency: A worldwide problem with health consequences. Am J ClinNutr. 357:266-281.

23. Holick MF, Chen TC. 2008. Vitamin D deficiency: A worldwide problem with health consequences. Am J Clin Nutr. 87:1080S-6S.

24. Horne JA, Östberg O. 1976. A self-assessment questionnaire to determine morningness-eveningness in human circadian rhythms. Int J Chronobiol. 4:97-110.

25. Humble MB. 2010. Vitamin D, light and mental health. J PhotochemPhotobiol B. 101:142-9.

26. Jääskeläinen T, Knekt P, Suvisaari, Männistö S, Partonen T, Sääksjärvi K, Kaartinen NE, Kanerva N, Lindfors O. 2015. Higher serum 25-hydroxyvitamin D concentrations are related to a reduced risk of depression. Br J Nutr. 113:1418-26.

27. Janssen CW, Lowry CA, Mehl MR, Allen JJ, Kelly KL, Gartner DE, Medrano A, Begay TK, Rentscher K, White JJ, et al. 2016. Whole-body hyperthermia for the treatment of major depressive disorder: a randomized clinical trial. JAMA Psychiatry. 73:789-95.

28. Jeong Jeong H, Moon E, Min Park J, Dae Lee B, Min Lee Y, Choi Y, In Chung Y. 2015. The relationship between chronotype and mood fluctuation in the general population. Psychiatry Res. 229:867-71.

29. Jussila A, Huotari-Orava R, Ylianttila L, Partonen T, Snellman E. 2016. Narrow-band ultraviolet $B$ radiation induces the expression of $\beta$-endorphin in human skin in vivo. $\mathrm{J}$ PhotochemPhotobiol B: Biology. 155:104-8.

30. Kanerva N, Kronholm E, Partonen T, Ovaskainen ML, Kaartinen NE, Konttinen H, Broms U, Männistö S. 2012. Tendency toward eveningness is associated with unhealthy dietary habits. Chronobiol Int. 29:920-7.

31. Kerkhof GA. 1985. Inter-individual differences in the human circadian system: a review. Biol Psychol. 20:83-112.

32. Kirnbauer R, Köck A, Neuner P, Förster E, Krutmann J, Urbanski A, Schauer E, Ansel JC, Schwarz T, Luger TA. 1991. Regulation of epidermal cell Interleukin-6 production by UV-light and corticosteroids. J Invest Dermatol. 96:484-9. 
33. Lemmer B. 2009. Discoveries of rhythms in human biological functions: a historical review. Chronobiol Int. 26:1019-68.

34. Leocadio-Miguel MA, Louzada FM, Duarte LL, Areas RP, Alam M, Freire MV, Fontenele-Araujo J, Menna-Barreto L, Pedrazzoli M. 2017. Latitudinal cline of chronotype. Sci Rep. 7:5437.

35. Liu Y, Ho R C-M, Mak A. 2012. Interleukin (IL)-6, tumor necrosis factor alpha (TNF$\alpha$ ) and soluble interleukin-2 reseptors (sIL-2R) are elevated in patients with major depressive disorder:A meta-analysis and meta-regression. J Affect Disord. 139:230-9.

36. Merikanto I, Lahti T, Kronholm E, Peltonen M, Laatikainen T, Vartiainen E, Salomaa V, Partonen T. 2013. Eveningtypes are prone to depression. ChronobiolInt. 30:719-25.

37. Miettinen ME, Kinnunen L, Leiviskä J, Keinänen-Kiukaannieni S, Korpi-Hyövälti E, Niskanen L, Oksa H, Saaristo T, Tuomilehto J, Vanhala M, et al. 2014. Association of serum 25-hydroxyvitamin D with lifestyle factors and metabolic and cardiovascular disease markers: Population-based cross-sectional study (FIN-D2D). PLoS One. 9:e100235.

38. Mondin TC, de Azevedo Cardoso T, Moreira FP, Wiener C, Oses JP, de Mattos Souza LD, Jansen K, da Silva Magalhães PV, Kapczinski F, da Silva RA. 2016. Circadian preferences, oxidative stress and inflammatory cytokines in bipolar disorder: A community study. J Neuroimmunol. 301:23-9.

39. Münch M, Bromundt V. 2012. Light and chronobiology: implications for health and disease. 14:448-53.

40. Murphy GM. Ultraviolet radiation and immunosuppression. 2009. Br J Dermatol. 161:90-5.

41. Randler C, Rahafar A. 2017. Latitude affects Morningness-Eveningness: evidence for the environment hypothesis based on a systematic review. Sci Rep. 7:39976.

42. Roenneberg T, Kumar CJ, Merrow M. 2007. The human circadian clock entrains to sun time. Curr Biol. 17:44-5.

43. Shattuck EC, Muehlenbein MP. 2015. Mood, behavior, testosterone, cortisol, and interleukin-6 in adults during immune activation: A pilot study to assess sickness behaviors in humans. Am J Human Biol. 27:133-5.

44. Shawa N, Rae DE, Roden LC. 2018. Impact of seasons on an individual's chronotype: current perspectives. Nat Sci Sleep. 10:345-54.

45. Skobowiat C, Dowdy JC, Sayre MR, Tuckey RC, Slominski A. 2011. Cutaneous hypothalamic-pituitary-adrenal axis homolog: regulation by ultraviolet radiation. Am J Physiol Endocrinol Metab. 301:E484-E93.

46. Skobowiat C, Slominski AT. 2015. UVB Activates Hypothalamic-Pituitary-Adrenal Axis in C57BL/6Mice. J Invest Dermatol. 135:1638-48. 
47. Slominski A, Wortsman J. 2000. Neuroendocrinology of the skin. Endocr Rev. 21:457-87.

48. Slominski AT, Kim TK, Shehabi HZ, Semak I, Tang EK, Nguyen MN, Benson HA, Korik E, Janjetovic Z, Chen J, et al. 2012. In vivo evidence for a novel pathway of vitamin D3 metabolism initiated by P450scc and modified by CYP27B1. FASEB J. 26:3901-15.

49. Urbanski A, Schwarz T, Neuner P, Krutmann J, Kirnbauer R, Luger TA. 1990. Ultraviolet Light Induces Increased Circulating Interleukin-6 in Human. J Invest Dermatol. 94:808-11.

50. Veleva BI, van Bezooijen RL, Chel VGM, Numans ME, Caliouw MAA. 2018. Effect of ultraviolet light on mood, depressive disorders and well-being. Photodermatol Photoimmunol Photomed. 34:288-97.

51. Vuolteenaho O. 1984. Beta-endorphin-related peptides in the human pituitary. Acta PhysiolScand Suppl. 531:1-84.

52. World Health Organization. International Agency for Research on Cancer. 2012. IARC Working Group on the Evaluation of Carcinogenic Risks to Humans. Radiation. IARC monogrEvalCarcinog Risks Hum. 100(Pt D):7-303.

53. Zeitzer JM, Khalsa SB, Boivin DB, Duffy JF, Shanahan TL, Kronauer RE, Czeisler CA. 2005. Temporal dynamics of late-night photic stimulation of the human circadian timing system. Am J Physiol Regul Integr Comp Physiol. 289:R839-44. 


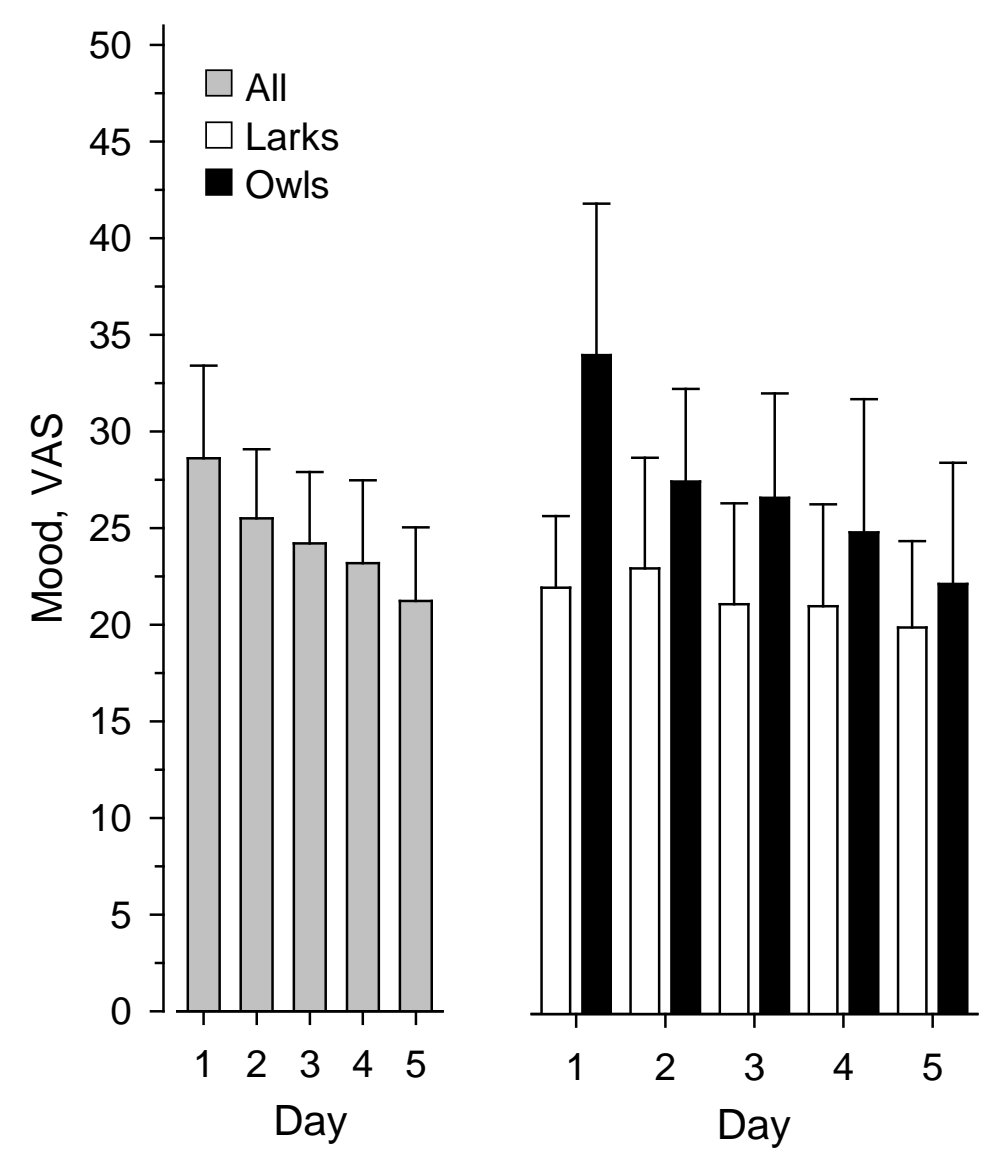

Figure 1. Perceived mood state immediately before NB UV-B irradiation on each day of the experiment and 24 hours after the last exposure. The bars depict the mean and SEM of the perceived mood of 11 participants on each day, taken all together (on the left, with gray bars), and by chronotype (Larks/Owls) (on the right, with white and black bars). The perceived mood state was evaluated as the mean of four dimensions of mood: 1) satisfaction, 2) tiredness, 3) wellbeing and 4) irritation. Each dimension was assessed on a 100-mm VAS scale, where a smaller value depicted a better mood. 

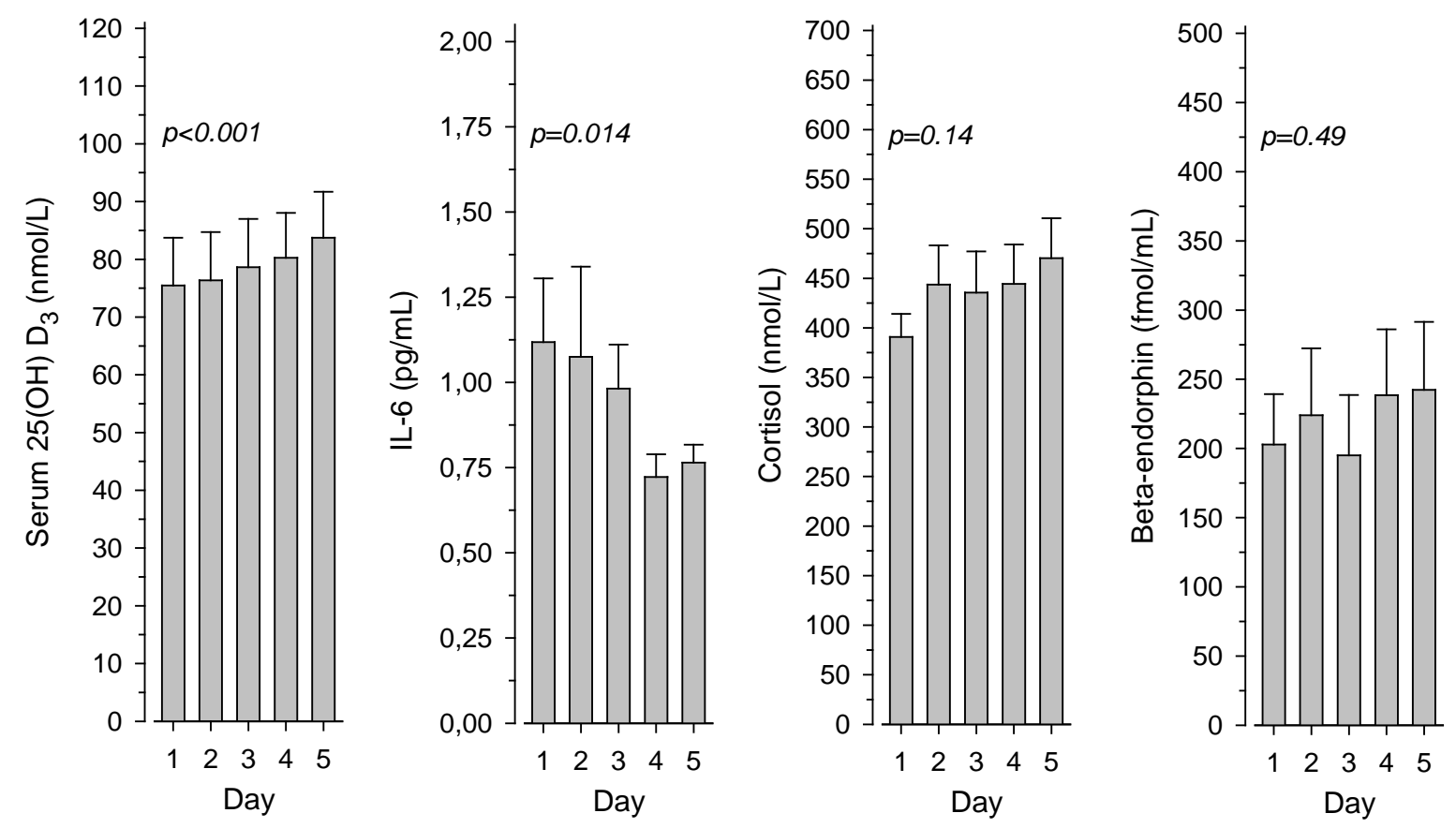

Figure 2a-d. The bars (from left to right) depict the means and SEM of a) serum 25(OH)D 3 (nmol/L), b) IL-6 (pg/mL), c) cortisol (nmol/L), and d) $\beta$-END (fmol/mL) on each day of the experiment. All blood samples were drawn immediately before the NB UVB exposures or 24 hours after the last exposure. p-values show linearity across the 5-day periods. 
Table 1. Mean serum vitamin D (25(OH) $\left.\mathrm{D}_{3}\right), \beta$-END, cortisol and IL-6 levels and mood VAS of the participants, grouped by chronotype (Larks/Owls).

\begin{tabular}{|c|c|c|c|c|c|}
\hline & Baseline & Day 2 & Day 3 & Day 4 & Day 5 \\
\hline \multicolumn{6}{|l|}{ “Larks" } \\
\hline $25(\mathrm{OH}) \mathrm{D}_{3}(\mathrm{nmol} / \mathrm{L})$ & $81.4 \pm 13.4$ & $81.4 \pm 14.1$ & $84.2 \pm 13.8$ & $85.0 \pm 13.0$ & $88.8 \pm 13.1$ \\
\hline$\beta$-END (fmol/mL) & $203 \pm 54.0$ & $278 \pm 84.0$ & $110 \pm 42.0$ & $225 \pm 73.0$ & $230 \pm 92.0$ \\
\hline Cortisol (nmol/L) & $364 \pm 34.0$ & $367 \pm 38.0$ & $332 \pm 47.0$ & $421 \pm 81.0$ & $421 \pm 81.0$ \\
\hline IL-6 (pg/mL) & $1.46 \pm 0.32$ & $1.56 \pm 0.56$ & $1.20 \pm 0.24$ & $0.83 \pm 0.10$ & $0.82 \pm 0.10$ \\
\hline VAS (mm) & $22.1 \pm 3.71$ & $23.1 \pm 5.73$ & $21.2 \pm 5.22$ & $21.1 \pm 5.27$ & $20.0 \pm 4.46$ \\
\hline \multicolumn{6}{|l|}{ "Owls" } \\
\hline $25(\mathrm{OH}) \mathrm{D}_{3}(\mathrm{nmol} / \mathrm{L})$ & $70.5 \pm 11.5$ & $72.2 \pm 11.2$ & $74.0 \pm 11.5$ & $76.3 \pm 10.5$ & $79.5 \pm 11.0$ \\
\hline$\beta$-END (fmol/mL) & $203 \pm 60.0$ & $179 \pm 63.0$ & $266 \pm 68.0$ & $250 \pm 76.0$ & $253 \pm 66.0$ \\
\hline Cortisol (nmol/L) & $413 \pm 34.0$ & $507 \pm 59.0$ & $522 \pm 43.0$ & $464 \pm 38.0$ & $467 \pm 48.0$ \\
\hline IL-6 (pg/mL) & $0.83 \pm 0.21$ & $0.67 \pm 0.10$ & $0.80 \pm 0.12$ & $0.63 \pm 0.09$ & $0.72 \pm 0.07$ \\
\hline VAS (mm) & $34.1 \pm 7.85$ & $27.5 \pm 4.80$ & $26.7 \pm 5.40$ & $24.9 \pm 6.89$ & $22.3 \pm 6.27$ \\
\hline
\end{tabular}

Absolute values are given as mean \pm SEM. 